\title{
DEMANDA DE POTÊNCIA DO VENTILADOR PARA AERAÇÃO DE MATERIAL EM COMPOSTAGEM
}

\author{
ANTONIO T. DE MATOS ${ }^{1}$, DENIS L. TEIXEIRA ${ }^{2}$, IVAN C. RIBEIRO ${ }^{3}$, \\ ED CARLO R. PAIVA ${ }^{4}$, ADÍLIO F. LACERDA FILHO ${ }^{5}$
}

\begin{abstract}
RESUMO: Neste trabalho, objetivou-se ajustar curvas de perda na pressão estática do ar, quando forçado em camadas de material orgânico, com diferentes espessuras, e dois estádios de degradação bioquímica, para possibilitar o cálculo da demanda de potência do sistema de ventilação. Os resíduos utilizados para a medição da perda de pressão do ar foram cama de frango misturada com casca dos frutos do cafeeiro, razão entre massas de 2,5:1, de forma a se obter relação C/N inicial de 30:1. Foi verificado que a perda na pressão estática do ar aumentou linearmente com a espessura da camada de composto orgânico e que houve aumento na perda de pressão estática com o aumento na vazão específica do ar, o que praticamente não foi alterado pelo estádio de degradação bioquímica do material em compostagem. Todos os modelos testados (potencial, logarítmico e quadrático) ajustaram-se bem aos dados experimentais, na faixa de vazão específica de ar entre 0,045 e $0,159 \mathrm{~m}^{3} \mathrm{~s}^{-1} \mathrm{~m}^{-2}$, podendo ser usado para predizer os gradientes de pressão estática no composto orgânico. A potência demandada pelo sistema de ventilação foi de $1,16 \mathrm{~W} \mathrm{t}^{-1}$ no material, antes da compostagem, e de 2,38 $\mathrm{W} \mathrm{t}^{-1}$, após 60 dias de compostagem, em camada com $1 \mathrm{~m}$ de espessura. Em camada de $2 \mathrm{~m}$ de espessura, a demanda foi de 17,70 e $21,65 \mathrm{~W} \mathrm{t}^{-1}$, respectivamente.
\end{abstract}

PALAVRAS-CHAVE: resíduos sólidos, leiras estáticas aeradas, processos aeróbios.

\section{VENTILATION POWER DEMAND FOR AERATION OF MATERIALS IN COMPOSTING}

\begin{abstract}
This study aimed to adjust loss curves in static air pressure when forced into layers of organic material, with different thicknesses and two stages of biochemical degradation, to enable the calculation of the power demand of the ventilation system. The residues used to evaluate air pressure loss were poultry litter mixed with coffee cherry husk, mass ratio of 2.5:1, to obtain an initial $\mathrm{C} / \mathrm{N}$ relation of 30:1. It was found that the loss in static pressure increased linearly with the thickness of the organic compound, and that there was an increase in static pressure loss with increased specific flow rate of air, which practically did not change by the biochemical degradation stage of the composting material. All models used (potential, logarithmic and quadratic) fit the experimental data in the range of specific air flow between 0.045 and $0.159 \mathrm{~m}^{3} \mathrm{~s}^{-1} \mathrm{~m}^{-2}$, and they may be used to predict the static pressure gradients in the organic compost. The power demanded by the ventilation system in layers of $1 \mathrm{~m}$ thickness was $1.16 \mathrm{~W} \mathrm{t}^{-1}$ in the material before composting, and $2.38 \mathrm{~W} \mathrm{t}^{-1}$, after 60 days of composting. In layers of $2 \mathrm{~m}$ thickness, the demand was 17.70 and $21.65 \mathrm{~W} \mathrm{t}^{-1}$, respectively.
\end{abstract}

KEYWORDS: solids residues, aerated static piles, aerobic process.

\footnotetext{
${ }^{1}$ Eng ${ }^{\mathrm{o}}$ Agrícola, Professor Associado, Departamento de Engenharia Agrícola, Universidade Federal de Viçosa, Viçosa - MG, atmatos@ufv.br, Bolsista do CNPq.

${ }^{2}$ Acadêmico do curso de Engenharia Agrícola e Ambiental, Departamento de Engenharia Agrícola, UFV,Viçosa - MG.

${ }_{3}^{3}$ Acadêmico do curso de Engenharia Agrícola e Ambiental, Departamento de Engenharia Agrícola, UFV,Viçosa - MG.

${ }^{4}$ Eng $^{\mathrm{o}}$ Civil, Professor, Departamento de Engenharia Civil, Universidade Federal de Goiás, Catalão - GO.

${ }^{5}$ Agrônomo, Professor Associado, Departamento de Engenharia Agrícola, UFV, Viçosa - MG.

Recebido pelo Conselho Editorial em: 16-2-2011

Aprovado pelo Conselho Editorial em: 10-2-2012
} 


\section{INTRODUÇÃO}

A compostagem é um sistema de reciclagem dos nutrientes e uma forma de acelerar a decomposição da matéria orgânica em relação ao que ocorreria no meio ambiente, melhorando as condições de atividade dos microrganismos (ORRICO JÚNIOR et al., 2009). É um dos meios mais eficientes para reciclagem de resíduos orgânicos para fins agrícolas (SATISHA \& DEVARAJAN, 2007; TORRES et al., 2007) e na eliminação de patógenos dos mesmos, além do baixo custo (COSTA et al., 2009). Nesse processo, ocorre a redução do volume inicial de resíduos, degradação de substâncias tóxicas e/ou patógenos, a matéria orgânica biodegradável é estabilizada, podendo ser manejada, estocada e aplicada como adubo orgânico, sem efeitos nocivos ao meio ambiente, desde que utilizada na dosagem adequada (ORRICO et al., 2007; ZHU, 2007).

Segundo PINTO (2001), a aeração do material em compostagem pode ser feita por revolvimento manual (processo Windrow) ou em camadas estáticas aeradas, sendo que, neste último, são utilizados ventiladores, sendo necessário o fornecimento de aproximadamente $1.200 \mathrm{~m} \mathrm{~h}^{-1} \mathrm{t}^{-1}$ de material orgânico seco em compostagem, demandando ventiladores de 370 a 1.500 W. HAUG (1993) recomendou, em relação ao material seco, a aplicação de 125 a $460 \mathrm{~m}^{3} \mathrm{~h}^{-1} \mathrm{t}^{-1}$, de forma a se obter o adequado fornecimento de oxigênio e a remoção do excesso de calor no material. PEREIRA NETO (2007), para atender à demanda de oxigênio, recomendou de 0,3 a $0,6 \mathrm{~m}^{3}$ de $\mathrm{O}_{2}$ para cada $\mathrm{kg}$ de sólidos voláteis. Considerando-se concentração de oxigênio no ar de $22 \%$ (em volume) e que a concentração de sólidos voláteis no material seja de $800 \mathrm{~g} \mathrm{~kg}^{-1}$, ter-seia uma faixa de recomendação de 71 a $142 \mathrm{~m}^{3} \mathrm{~h}^{-1}$ de ar para cada tonelada de material orgânico seco.

Considerando-se que os custos de aquisição e operação do ventilador estão, diretamente, relacionados com sua potência, e que esta é função da vazão de ar, da pressão estática e da eficiência do sistema de ventilação, torna-se importante o estudo dos fatores que influenciam na demanda de potência do equipamento. Essa pressão é determinada, convencionalmente, por meio de equações ou gráficos que relacionam a perda de pressão por unidade de espessura da camada do produto (gradiente de pressão) com a vazão específica do ar (vazão do ar por unidade de área).

SILVA et al. (2008) citaram que a perda de pressão devida à passagem do ar através de qualquer produto depende da vazão específica, forma e características do produto, do número, tamanho e configuração dos vazios e da espessura da camada de produto.

Modelos matemáticos são utilizados para predizer a perda de pressão em fluidos forçados através de materiais porosos, tal como composto orgânico (McGUCKIN et al., 1999). SILVA et al. (2008) afirmaram que a perda de pressão em produtos agrícolas pode ser representada por curvas potenciais (Equação de Shedd) e logarítmicas (Equação de Hukill \& Ives), conforme as eq.(1) e (2):

$$
\Delta \mathrm{P}=\mathrm{A} 1 \mathrm{Q}_{\mathrm{a}}^{\mathrm{B} 1}
$$

$$
\Delta \mathrm{P}=\frac{\mathrm{A} 2 \mathrm{Q}_{\mathrm{a}}{ }^{2}}{\ln \left(1+\mathrm{B} 2 \mathrm{Q}_{\mathrm{a}}\right)}
$$

em que,

$\mathrm{Q}_{\mathrm{a}}$ - vazão específica de $\operatorname{ar}\left(\mathrm{L}^{3} \mathrm{~T}^{-1} \mathrm{~L}^{-2}\right)$;

$\Delta \mathrm{P}$ - gradiente de pressão estática $\left(\mathrm{M} \mathrm{T}^{-2} \mathrm{~L}^{-2}\right)$, e

A1, A2, B1 e B2 - parâmetros obtidos no ajuste dos modelos aos dados experimentais.

McGUCKIN et al. (1999) apresentaram um modelo matemático quadrático, proposto por Ergun, para estimar a perda na pressão de ar através de uma camada de material orgânico (eq.(3)):

$$
\Delta \mathrm{P}=\mathrm{A} 3 \quad \mathrm{Q}_{\mathrm{a}}+\mathrm{B} 3 \mathrm{Q}_{\mathrm{a}}{ }^{2}
$$

Os parâmetros $A 3$ e $B 3$ estão associados às perdas de energia viscosa e cinética, respectivamente. Em escoamento turbulento, sob alta vazão específica de ar, a componente cinética é a principal fonte de perda de energia no sistema. Considera-se que a perda na pressão varie 
linearmente com a espessura da camada de material, podendo ser obtida utilizando-se da Equação 4 para o cálculo da pressão estática a ser fornecida pelo ventilador:

$$
\mathrm{P}=\Delta \mathrm{PH}
$$

em que,

$\mathrm{P}$ - perda de pressão devido à resistência do material $\left(\mathrm{M} \mathrm{T}^{-2} \mathrm{~L}^{-1}\right), \mathrm{e}$

$\mathrm{H}$ - altura de material, L.

Neste trabalho, objetivou-se ajustar curvas de perda na pressão estática do ar, quando forçado em camadas de material orgânico, com diferentes espessuras, e dois estádios de degradação bioquímica, para possibilitar o cálculo da demanda de potência do sistema de ventilação.

\section{MATERIAL E MÉTODOS}

O experimento foi conduzido na Área Experimental de Hidráulica, Irrigação e Drenagem, Departamento de Engenharia Agrícola - UFV, Viçosa, Estado de Minas Gerias. Os resíduos utilizados para a medição da perda de pressão do ar foram cama de frango misturada com casca de frutos do cafeeiro, na proporção (em massa) de 2,5:1, de forma que se possibilitasse obter relação $\mathrm{C} / \mathrm{N}$ inicial 30:1. Após efetuada a mistura dos materiais, verificou-se que a relação $\mathrm{C} / \mathrm{N}$ inicial variou entre 20:1 e 30:1, devido à natural heterogeneidade dos mesmos e de sua mistura. $\mathrm{O}$ material, depois de submetido à compostagem em sistema de leiras estáticas aeradas, durante 60 dias, foi submetido às mesmas medições de perda de pressão do ar nele forçado. Optou-se por não efetuar estratificação granulométrica do material, com eliminação dos "finos" por peneiramento, por se entender que isso seria uma interferência que não ocorreria, normalmente, em condições normais de operação do sistema. Tanto o material recém-misturado como o submetido à compostagem apresentaram diâmetro de partículas menor que $6 \mathrm{~mm}$.

Nos resíduos foram quantificados o teor de água, por gravimetria, e a massa específica, a qual foi obtida dividindo-se a massa da camada de $1 \mathrm{~m}$ de espessura de material, cuja forma de acondicionamento está descrita a seguir (Figura 1), pelo volume por ela ocupado na coluna. As características físicas dos materiais estão apresentadas na Tabela 1.

TABELA 1. Características físicas da casca de frutos do cafeeiro misturada com cama de frango. Physical characteristics of the bark of coffee cherries mixed with poultry litter.

\begin{tabular}{lcc}
\hline Condição do Material & $\begin{array}{c}\text { Teor de Água (b.u.) } \\
\left(\mathrm{g} \mathrm{kg}^{-1}\right)\end{array}$ & $\begin{array}{c}\text { Massa Específica } \\
\left(\mathrm{kg} \mathrm{m}^{-3}\right)\end{array}$ \\
\hline Antes do início da compostagem & 627,1 & 382 \\
Após a compostagem & 622,4 & 577 \\
\hline
\end{tabular}

Para a medição da perda de pressão através da massa de material, foi utilizado um protótipo conforme ilustrado na Figura 1, constituído pelos seguintes componentes: (1) coluna de chapa galvanizada, medindo $1,0 \mathrm{~m}$ de altura, com $0,30 \mathrm{~m}$ de diâmetro; (2) tomadas para medição da pressão estática, representadas por sete tubos de cobre ( $5 \mathrm{~mm}$ de diâmetro), distanciados em $0,10 \mathrm{~m}$ no sentido vertical, em torno da coluna; (3) piso perfurado, constituído por chapa com furos circulares; (4) câmara plenum, em chapa galvanizada, cujas dimensões são, aproximadamente: 0,52 m (comprimento), 0,47 m (largura) e 0,40 m de altura; (5) tubo de PVC, medindo 2,0 m de comprimento e diâmetro de $0,20 \mathrm{~m}$, utilizado para insuflar o ar do ventilador até o plenum; (6) homogeneizador, para uniformização do fluxo de ar; (7) ventilador centrífugo de pás retas, acionado por motor elétrico com potência de $735 \mathrm{~W}$ em $3.415 \mathrm{rpm}$; (8) diafragma fixo à entrada de ar, para possibilitar a variação na vazão.

O enchimento da coluna de chapa galvanizada foi realizado, colocando-se sucessivas camadas do material com espessura de $0,20 \mathrm{~m}$, seguindo-se o período de uma noite para assentamento de 
nova camada de material até ser completada a espessura de $1 \mathrm{~m}$, sendo realizadas as medidas de pressão estática na posição vertical de $1 \mathrm{~m}$.

1 - coluna de chapa galvanizada

2 - tomadas para medição da pressão estática

3 - piso perfurado

4 - câmara plenum

5 - tubo de PVC

6 - homogeneizador do escoamento do ar

7 - ventilador centrífugo

8 - diafragma

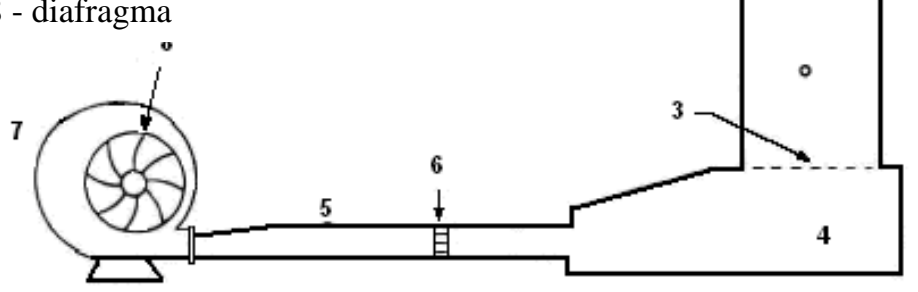

FIGURA 1. Croqui: esquema de protótipo utilizado para a determinação do gradiente de pressão estática em diferentes vazões específicas de ar. Sketch: prototype scheme used for determining the gradient of static pressure at different specific flow rates of air.

Mediu-se a pressão estática na câmara plenum, utilizando-se de um manômetro digital marca KIMO, modelo - MP 120, com resolução de 0,1 mmca. Para medir a velocidade do ar, utilizou-se de um anemômetro de pás, marca - PROVA, modelo - AVM 07, com resolução de $0,01 \mathrm{~m} \mathrm{~s}^{-1}$. A vazão de ar foi obtida pelo resultado do produto entre a velocidade média do ar e a área da seção transversal do duto de saída. Durante as medidas da velocidade do ar, para atingir a sensibilidade de medida do aparelho, utilizou-se um cone que, condicionando a passagem do ar por uma seção de menor diâmetro, proporcionou aumento da velocidade do ar, de forma a conferir maior sensibilidade ao anemômetro nele posicionado.

As medições de perda de pressão estática, em diferentes posições na camada de material, foram feitas com três repetições, no plenum e a cada $0,10 \mathrm{~m}$ de espessura na coluna, em ambos os materiais analisados. As vazões específicas utilizadas variaram de 0,045 a $0,159 \mathrm{~m}^{3} \mathrm{~s}^{-1} \mathrm{~m}^{-2}$.

Utilizando-se da regressão linear, foram ajustadas equações de perda de pressão como função da profundidade da camada de material. Além disso, foram ajustados os modelos potencial (eq.(1)), logarítmico (eq.(2)) e quadrático (eq.(3)) de gradiente de pressão (obtido com dados de perda de pressão para $1 \mathrm{~m}$ de coluna de material) como função da vazão específica de ar. Para ajuste das equações, foi utilizado o programa Statistica for Windows Release 5.0.

Com base nas equações ajustadas, foram calculadas as potências absorvidas pelo ventilador, utilizando-se da eq.(5), no caso da aeração de pilhas de material antes do início e após a compostagem.

$$
\mathrm{Pot}=\frac{\mathrm{PQ}}{\eta}
$$

em que,

Pot - potência elétrica absorvida pelo ventilador, W;

$\mathrm{P}$ - pressão total fornecida pelo ventilador, $\mathrm{Pa}$;

$\mathrm{Q}$ - vazão de ar, $\mathrm{m}^{3} \mathrm{~s}^{-1}$, e

$\eta$ - rendimento do ventilador, adimensional. 


\section{RESULTADOS E DISCUSSÃO}

Avaliando-se os valores obtidos para as massas específicas dos materiais na condição massa fresca (úmidos), verificou-se que a casca de frutos do cafeeiro misturada com cama de frango, antes de terem sido submetidos à compostagem, ficou próximo ao que foi obtido por SILVA et al. (2008), depois de decorridos 30 dias de compostagem de bagaço de cana-de-açúcar picado, misturado com cama de frango, ocasião em que a massa específica era de $403 \mathrm{~kg} \mathrm{~m}^{-3}$. Os mesmos autores observaram que, decorridos 90 dias, a massa específica era de $424 \mathrm{~kg} \mathrm{~m}^{-3}$ e, ao final do período de compostagem, de $577 \mathrm{~kg} \mathrm{~m}^{-3}$. Segundo os autores, o aumento na massa específica do material, com o tempo de degradação bioquímica, foi resultante da diminuição no tamanho das partículas, em decorrência da referida decomposição microbiana do material. Segundo PEREIRA NETO (2007), o composto orgânico deve ter massa específica úmida entre 450 e $550 \mathrm{~kg} \mathrm{~m}^{-3}$.

Nas Figuras 2 e 3, estão apresentadas, respectivamente, as curvas de perda de pressão do ar em escoamento, como função da espessura da camada de casca de frutos do cafeeiro misturada com cama de frango, antes do início da compostagem e após o material ter sido compostado.

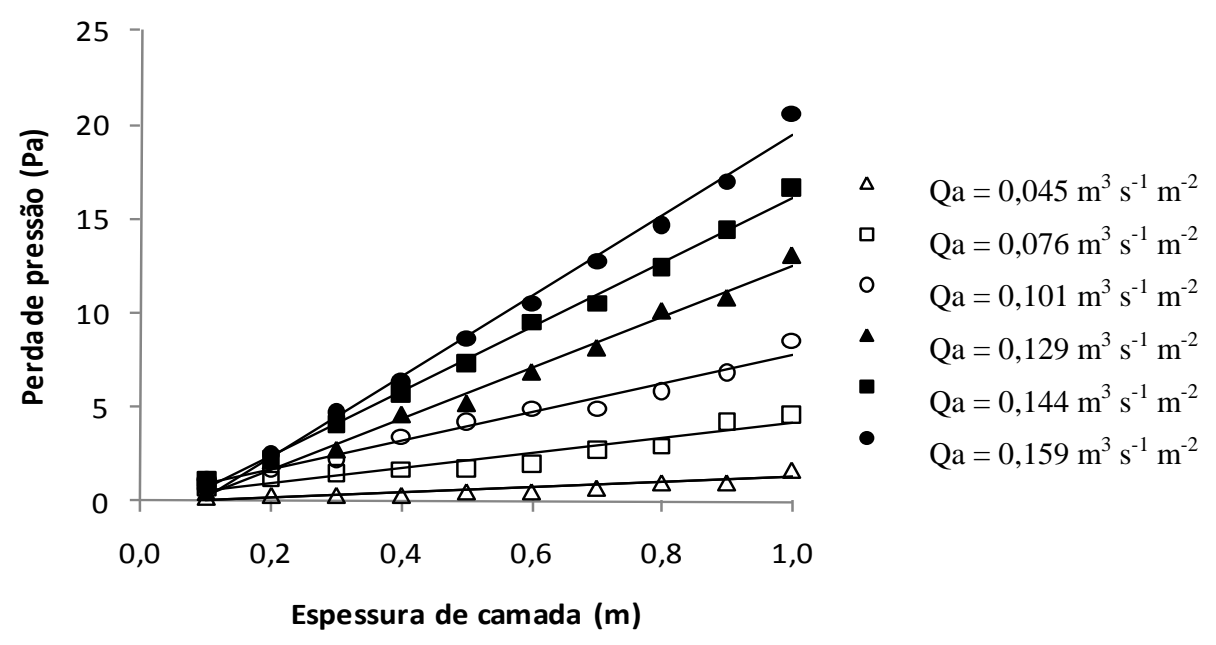

FIGURA 2. Variação na perda de pressão do ar em coluna de casca dos frutos do cafeeiro, misturada com cama de frango, avaliada antes do início do processo de compostagem, sob diferentes vazões específicas. Variation in the loss of air pressure in column of the coffee cherry bark mixed with chicken litter evaluated before the start of the composting process under different specific flow rates.

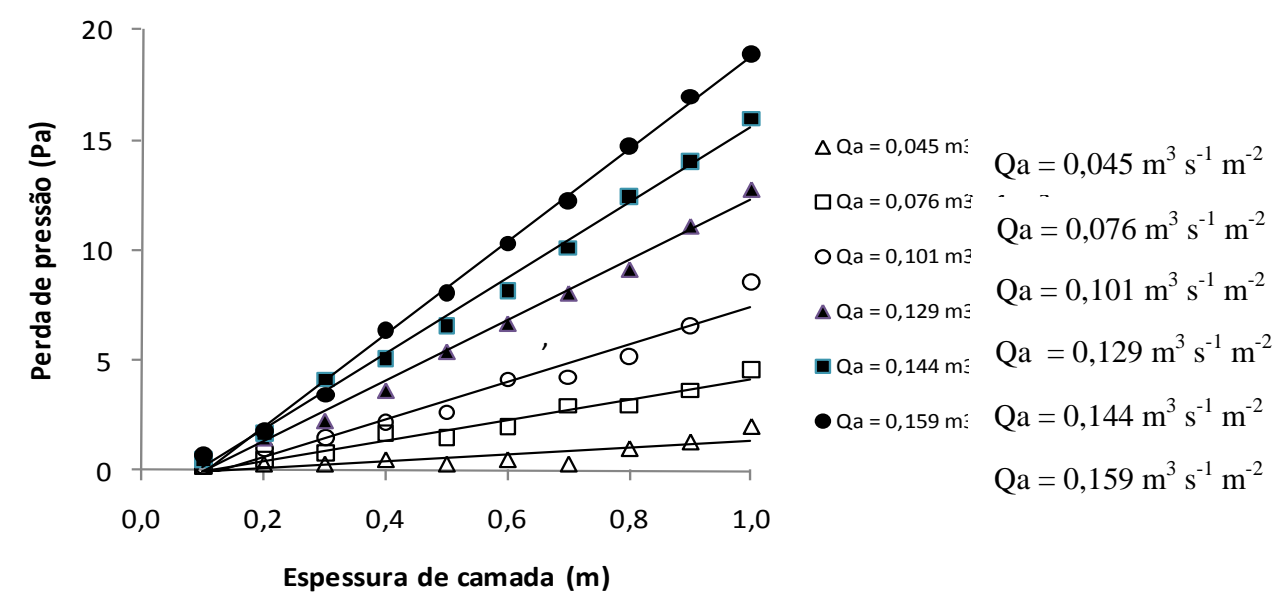

FIGURA 3. Variação na perda de pressão do ar em coluna de casca dos frutos do cafeeiro, misturada com cama de frango, avaliada ao final do período de compostagem, sob diferentes vazões específicas. Variation in the loss of air pressure in column of the coffee cherry bark mixed with chicken litter assessed at the end of the period of composting under different specific flow rates. 
Verificou-se aumento linear da perda de pressão de ar com a espessura da camada de material, tanto naquele em que o processo de compostagem não ocorreu, como naquele em que ocorreu, por onde o ar foi forçado a escoar. Além disso, verificou-se aumento na declividade da curva, ou seja, maior gradiente de pressão com o aumento na vazão específica de ar.

Nas Tabelas 2 e 3, estão apresentadas as curvas ajustadas e os respectivos coeficientes de determinação $\left(\mathrm{R}^{2}\right)$ para perda de pressão como função da espessura da camada de material.

TABELA 2. Equações ajustadas para perda na pressão $(\mathrm{P})$ como função da espessura $(\mathrm{H})$ da camada de cascas de frutos do cafeeiro, misturada com cama de frango, antes do início do processo de compostagem, para diferentes vazões específicas $\left(Q_{a}\right)$. Equations adjusted for loss in pressure $(\mathrm{P})$ as a function of depth $(\mathrm{H})$ of coffee cherry bark mixed with chicken litter before the beginning of the composting process for different specific flow rates $(\mathbf{Q})$.

\begin{tabular}{ccc}
\hline $\mathrm{Q}_{\mathrm{a}}\left(\mathrm{m}^{3} \mathrm{~s}^{-1} \mathrm{~m}^{-2}\right)$ & Equação & $\mathrm{R}^{2}$ \\
\hline 0,045 & $\mathrm{P}=1,3726 \mathrm{H}-0,1176$ & 0,823 \\
0,076 & $\mathrm{P}=4,0286 \mathrm{H}+0,1176$ & 0,914 \\
0,101 & $\mathrm{P}=7,6234 \mathrm{H}+0,1895$ & 0,974 \\
0,129 & $\mathrm{P}=13,476 \mathrm{H}-1,0000$ & 0,991 \\
0,144 & $\mathrm{P}=17,136 \mathrm{H}-1,0135$ & 0,996 \\
0,159 & $\mathrm{P}=21,313 \mathrm{H}-1,8889$ & 0,994 \\
\hline
\end{tabular}

Em que, $P$ está expresso em Pa e $H$ em m.

TABELA 3. Equações ajustadas para perda na pressão $(\mathrm{P})$ como função da espessura $(\mathrm{H})$ da camada de cascas de fruto do cafeeiro, misturada com cama de frango, ao final do processo de compostagem, para diferentes vazões específicas $\left(Q_{a}\right)$. Equations adjusted for loss in pressure $(\mathrm{P})$ as a function of the depth $(\mathrm{H})$ of the coffee cherry bark mixed with chicken litter at the end of the composting process for different specific flow rates (Qa).

\begin{tabular}{ccc}
\hline $\mathrm{Q}_{\mathrm{a}}\left(\mathrm{m}^{3} \mathrm{~s}^{-1} \mathrm{~m}^{-2}\right)$ & Equação & $\mathrm{R}^{2}$ \\
\hline 0,045 & $\mathrm{P}=1,5627 \mathrm{H}-0,2026$ & 0,676 \\
0,076 & $\mathrm{P}=4,6524 \mathrm{H}-0,4902$ & 0,961 \\
0,101 & $\mathrm{P}=8,5087 \mathrm{H}-1,0719$ & 0,959 \\
0,129 & $\mathrm{P}=13,720 \mathrm{H}-1,4574$ & 0,993 \\
0,144 & $\mathrm{P}=17,219 \mathrm{H}-1,6079$ & 0,994 \\
0,159 & $\mathrm{P}=21,016 \mathrm{H}-2,2157$ & 0,996 \\
\hline
\end{tabular}

Em que, $P$ está expresso em Pa e $H$ em $\mathrm{m}$.

Verifica-se, com base nas equações ajustadas, comportamento semelhante dos dois materiais, independentemente do seu estádio de degradação bioquímica. Embora o material compostado tenha apresentado teor de água semelhante e massa específica cerca de 50\% maior (Tabela 1), ele não proporcionou aumento na perda de pressão do ar. Acredita-se que isso se deva à alteração no formato das partículas de material orgânico com sua degradação bioquímica, proporcionando o aparecimento de estrutura mais granular, de menor resistência ao escoamento do ar (SILVA et al., 2008). MOREIRA et al. (2008) encontraram, no entanto, para mesma vazão específica e espessura de camada de material, aumento nas perdas de pressão do ar com o tempo de compostagem do material. Além disso, esses autores, também de forma diferente, obtiveram melhor ajuste de equações logarítmicas para relacionar a perda de pressão e a espessura da camada de material. JEKAYINFA (2006), avaliando a resistência ao escoamento do ar em sementes de alfarroba, quando submetidas à vazão específica de ar variando entre $0,201 \mathrm{e} 0,275 \mathrm{~m}^{3} \mathrm{~s}^{-1} \mathrm{~m}^{-2}$, em colunas de material com espessura de 76,5 e 382,5 $\mathrm{mm}$ e teor de água (base úmida) variando entre e 95,0 e $376,5 \mathrm{~g} \mathrm{~kg}^{-1}$, verificou que a resistência ao escoamento do ar diminuiu com a diminuição da vazão 
específica aplicada, com a espessura da camada de material e com o aumento no teor de água das sementes. NICOLAI \& JANI (2001) verificaram que a perda na pressão do ar aumentou quando se tornou maior a percentagem de composto orgânico misturado às raspas de madeira (granulometria entre 1,3 e 7,6 cm), e os autores encontraram perda de carga entre 4 e $14 \mathrm{~Pa}$ no material, quando submetido à vazão específica de $234 \mathrm{~m}^{3} \mathrm{~h}^{-1} \mathrm{~m}^{-2}$, em biofiltros utilizados no controle de odor em suinoculturas. Verificaram, também, que o aumento no teor de água do composto orgânico misturado proporcionou aumento na perda de pressão do ar.

Depois de realizadas as devidas transformações nos dados, os valores de gradiente de pressão foram plotados como função da vazão específica (Figura 4).

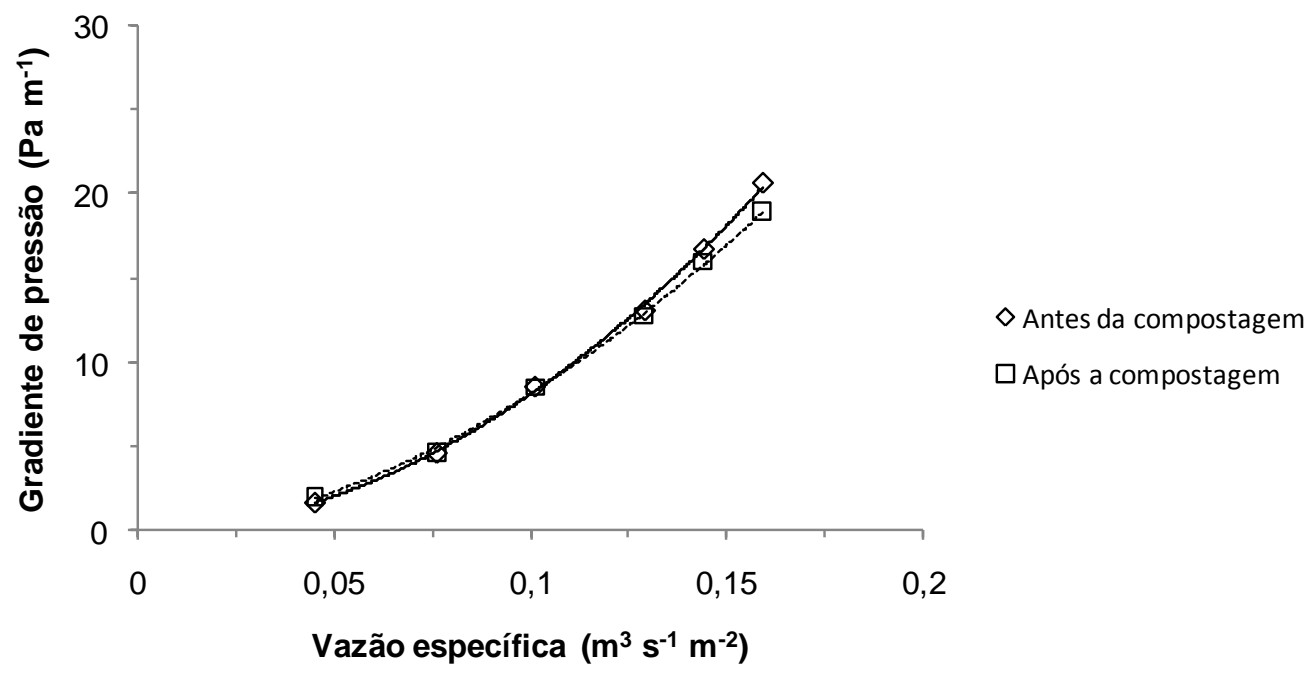

FIGURA 4. Gradiente de pressão do ar em coluna de cascas de frutos do cafeeiro misturada com cama de frango, antes e após a compostagem, em função da vazão específica. Gradient of air pressure in column of coffee cherry bark mixed with chicken litter, before and after composting, depending on the specific flow.

Com base no comportamento dos dados experimentais obtidos, pode-se verificar que, à medida que foi aumentada a vazão específica de ar insuflado na camada de material, aumentou o gradiente de pressão do ar. Entretanto, avaliando as curvas obtidas para os materiais em diferentes estádios de degradação bioquímica, verifica-se haver grande proximidade entre elas, não se distinguindo variações para vazões específicas menores que $0,125 \mathrm{~m}^{3} \mathrm{~s}^{-1} \mathrm{~m}^{-2}$. Entende-se inclusive que, na compostagem de resíduos orgânicos em leiras estáticas aeradas, essas diferenças no gradiente de pressão possam ser negligenciadas para pequenas vazões específicas aplicadas (até $0,16 \mathrm{~m}^{3} \mathrm{~s}^{-1} \mathrm{~m}^{-2}$ ) e quando a massa específica do material não for muito diferente de $500 \mathrm{~kg} \mathrm{~m}^{-3}$.

De forma diferente do que foi observado neste trabalho, SILVA et al. (2008) obtiveram aumento no gradiente de pressão estática do ar com o tempo de maturação do material, considerando-se a mesma vazão específica de ar. Segundo os autores, isso se deve ao aumento na massa específica do material; entretanto, mesmo tendo ocorrido também neste trabalho, o aumento na massa específica do material não proporcionou aumento na resistência à passagem do ar, o que pode estar associado à granularização do material. SILVA et al. (2008) obtiveram gradientes de pressão de 10 a $20 \mathrm{~Pa} \mathrm{~m}^{-1}$ em bagaço de cana-de-açúcar picado, misturado com cama de frango, antes de terem sido submetidos à compostagem, quando utilizaram vazão específica de ar entre 0,6 e $2,5 \mathrm{~m}^{3} \mathrm{~s}^{-1} \mathrm{~m}^{-2}$. No material compostado, esses autores observaram gradiente de pressão próximo a $180 \mathrm{~Pa} \mathrm{~m}^{-1}$ quando o material foi submetido à vazão específica de $0,2 \mathrm{~m}^{3} \mathrm{~s}^{-1} \mathrm{~m}^{-2}$. McGUCKIN et al. (1999) encontraram gradientes de pressão entre 3 e $120 \mathrm{~Pa} \mathrm{~m}^{-1}$ para vazão específica entre 0,007 e $0,04 \mathrm{~m}^{3} \mathrm{~s}^{-1} \mathrm{~m}^{-2}$, em mistura de resíduos de alface, cebola, dejeto de frango e serragem de madeira, na proporção de 101:130:52:1 (matéria fresca). KUSIŃSKA (2008), avaliando a resistência ao escoamento do ar através de grãos de trigo, verificou que o aumento da densidade dos grãos de trigo 
de 787 a $1.063 \mathrm{~kg} \mathrm{~m}^{-3}$ e o aumento da vazão específica do ar de 0,15 a $0,5 \mathrm{~m}^{3} \mathrm{~s}^{-1} \mathrm{~m}^{-2}$ proporcionaram aumento no gradiente de pressão de 0,73 para 9,24 $\mathrm{Pa} \mathrm{m}^{-1}$.

$\mathrm{Na}$ Tabela 4, estão apresentadas as equações ajustadas para a estimativa do gradiente de pressão estática como função da vazão específica, para os modelos potencial (eq.(1)), logarítmico (eq.(2)) e quadrático (eq.(3)).

TABELA 4. Equações ajustadas e coeficientes de determinação obtidos para gradiente de pressão em função da vazão específica do ar forçado em colunas de cascas de fruto do cafeeiro, misturadas com cama de frango, antes e após o processo de compostagem. Adjusted equations and determination coefficients obtained for gradient of air pressure as function of the specific flow rate of the air forced through coffee cherry bark mixed with chicken litter columns, before and after the composting process.

\begin{tabular}{cllc}
\hline \multicolumn{1}{c}{ Material } & \multicolumn{1}{c}{ Modelo } & \multicolumn{1}{c}{ Equação } & $\mathrm{R}^{2}$ \\
\hline \multirow{4}{*}{ Antes da compostagem } & Potencial (Shedd) & $\Delta \mathrm{P}=0,5317 \mathrm{Q}_{\mathrm{a}}{ }^{1,8161}$ & 0,999 \\
& Logarítmico (Hukill \& Yves) $\Delta \mathrm{P}=\frac{14,8585 \mathrm{Q}_{\mathrm{a}}{ }^{2}}{\ln \left(1+7,062210^{8} \mathrm{Q}_{\mathrm{a}}\right)}$ & 0,999 \\
& Quadrático (Ergun) & $\Delta \mathrm{P}=-5,310^{5} \mathrm{Q}_{\mathrm{a}}+0,8073 \mathrm{Q}_{\mathrm{a}}{ }^{2}$ & 0,999 \\
\hline \multirow{2}{*}{ Após a compostagem } & Potencial (Shedd) & $\Delta \mathrm{P}=0,7821 \mathrm{Q}_{\mathrm{a}}{ }^{1,9849}$ & 0,999 \\
& Logarítmico (Hukill \& Yves) $\Delta \mathrm{P}=\frac{4,8911 \mathrm{Q}_{\mathrm{a}}{ }^{2}}{\ln \left(1+4248,787 \mathrm{Q}_{\mathrm{a}}\right)}$ & 0,999 \\
& Quadrático (Ergun) & $\Delta \mathrm{P}=1,371810^{-2} \mathrm{Q}_{\mathrm{a}}+0,6673 \mathrm{Q}_{\mathrm{a}}{ }^{2}$ & 0,999 \\
\hline
\end{tabular}

Em que $Q_{a}$ expresso em $\mathrm{m}^{3} \mathrm{~s}^{-1} \mathrm{~m}^{-2} \mathrm{e} \Delta P$ em $\mathrm{kPa} \mathrm{m}^{-1}$.

Poucas são as informações disponíveis de gradiente de pressão estática do ar como função da vazão específica do ar para materiais diferentes de grãos. Procurou-se, então, comparar os parâmetros ajustados neste trabalho com os obtidos em ensaios em colunas de resíduos orgânicos misturados (McGUCKIN et al., 1999; SILVA et al., 2008). McGUCKIN et al. (1999), ao ajustarem equações para relacionar gradiente de pressão e vazão específica em mistura de resíduos de alface, cebola, dejeto de frango e serragem de madeira (na proporção em termos de massa fresca, de 101:130:52:1), encontraram, para o modelo potencial, $\mathrm{A} 1=3,31$ e B1 = 1,08; logarítmico, $\mathrm{A} 2=$ 13,6 e B2 $=5,85$; e quadrático, A3 $=2,34$ e B3 $=6,37$. SILVA et al. (2008) obtiveram, em bagaço de cana-de-açúcar picado, $\mathrm{A} 1=5,248 \times 10^{-4}$ e $\mathrm{B} 1=2,427, \mathrm{~A} 2=9,87 \times 10^{-3}$ e $\mathrm{B} 2=22,935$, no mesmo material, depois de misturado com cama de frango e com 30 dias de compostagem, $\mathrm{A} 1=6,48 \times 10^{-4}$, $\mathrm{B} 1=2,427 ; \mathrm{A} 2=0,136$ e B2 $=546,104$.

Com base nos parâmetros ajustados para o modelo potencial, verificou-se maior aproximação dos parâmetros ajustados neste trabalho com os obtidos por McGUCKIN et al. (1999). Comparando-se os parâmetros ajustados para a equação logarítmica, verificou-se maior diferença nos valores de $B 2$, porém esse coeficiente tem pequena influência nos valores calculados de gradiente de pressão e, dessa forma, pode-se considerar que a equação ajustada por McGUCKIN et al. (1999) também foi a que mais se aproximou das obtidas neste trabalho. Acredita-se que a introdução de agentes sintéticos para aumentar a macroporosidade do material, efetuada pelos referidos autores, tenha proporcionado condições de escoamento do ar semelhantes às obtidas no material avaliado neste estudo, proporcionado pela mistura de cascas de fruto do cafeeiro. Ao contrário do que se esperava, já que em relação aos outros modelos houve boa aproximação das equações, os parâmetros ajustados para a equação quadrática, obtidos neste trabalho, foram menores do que aqueles obtidos por McGUCKIN et al. (1999). Talvez isso possa ser indicativo da inadequabilidade da utilização desse tipo de modelo para relacionar gradiente de perda de pressão e vazão específica.

Considerando a aplicação da vazão específica de ar de $1.200 \mathrm{~m}^{3} \mathrm{~h}^{-1} \mathrm{t}^{-1}$ (PINTO, 2001) e calculando-se a potência demandada (considerando-se rendimento de $60 \%$ do ventilador) para 
aeração do material orgânico estudado neste trabalho ( $1 \mathrm{~m}$ de espessura da camada), foram obtidos, respectivamente, os valores de $1,2 \mathrm{~W} \mathrm{t}^{-1}$ para o material antes de ser submetido à compostagem, e de $2,4 \mathrm{~W} \mathrm{t}^{-1}$ para o material compostado. Caso se utilizem $2 \mathrm{~m}$ de espessura de camada de material, as potências demandadas passam a ser, respectivamente, de 17,7 e $21,7 \mathrm{~W} \mathrm{t}^{-1}$. Considerando a vazão específica recomendada por PEREIRA NETO (2007), que é de 71 a $142 \mathrm{~m}^{3} \mathrm{~h}^{-1} \mathrm{t}^{-1}$, ou seja, de 8 a 17 vezes inferior à recomendada por PINTO (2001), ter-se-ia uma potência demandada variando de 0,001 a $0,003 \mathrm{~W} \mathrm{t}^{-1}$, o que proporcionaria uma potência de 400 a 800 vezes menor.

Os valores obtidos são inferiores aos apresentados por MOREIRA et al. (2008), que trabalharam com mistura de bagaço de cana-de-açúcar picado (relação $\mathrm{C} / \mathrm{N}$ inicial de 30:1), considerando-se vazão de $1.200 \mathrm{~m}^{3} \mathrm{~h}^{-1} \mathrm{t}^{-1}$, em que obtiveram, para aeração de $1 \mathrm{~m}$ de espessura da camada de material com 30 dias de compostagem, potência demandada de 44,2 $\mathrm{W} \mathrm{t}^{-1}$. Em camada com espessura de $2,0 \mathrm{~m}$, a potência demandada passou para $63,3 \mathrm{~W} \mathrm{t}^{-1}$. Com o material submetido a 90 dias de compostagem, os autores informaram que seriam exigidos $278 \mathrm{~W} \mathrm{t}^{-1}$, para $1,0 \mathrm{~m}$ de espessura de camada e $361 \mathrm{~W} \mathrm{t}^{-1}$, para $2,0 \mathrm{~m}$. Esses valores estão abaixo do citado por PINTO (2001) ao se referir à compostagem aerada de lodo de esgoto, que foi de $820,6 \mathrm{~W} \mathrm{t}^{-1}\left(1,1 \mathrm{HP} \mathrm{t}^{-1}\right)$.

A variabilidade dos valores encontrados, considerando os diferentes autores, sugere que o valor da potência demandada pelo ventilador deva variar com o tipo de resíduo e suas características (tamanho, forma, teor de água), tal como reportado por JEKAYINFA (2006), e com os fatores ambientais, como temperatura ambiente, umidade relativa do ar, dentre outros. Além disso, deve-se levar em consideração se a demanda de ar será para suprir a demanda de oxigênio, controlar a temperatura da leira ou para a remoção de água, sendo esse último um referencial muito comum em compostagem de lodo de esgoto (HAUG, 1993; PINTO, 2001). INÁCIO \& MILLER (2009) citaram que a demanda de ar para controlar a temperatura na leira é cerca de 9 vezes maior que a necessária para suprir a demanda de oxigênio.

\section{CONCLUSÕES}

A perda na pressão estática do ar aumentou linearmente com a espessura da camada de composto orgânico.

Houve aumento no gradiente de pressão estática do ar com o aumento na vazão específica do ar, tendo os materiais, avaliados antes e após a compostagem, apresentando comportamentos semelhantes.

Todos os modelos matemáticos utilizados ajustaram-se bem aos dados experimentais, na faixa de vazão específica de ar investigada, podendo ser utilizados para predizer os gradientes de pressão estática no composto orgânico. Entretanto, recomenda-se o uso do modelo potencial, por ser simples e de uso comum para descrever o gradiente de pressão como função da vazão específica.

A potência demandada pelo sistema de ventilação do material orgânico foi baixa, sendo de $1,2 \mathrm{~W} \mathrm{t}^{-1}$, no material antes de ter sido submetido à compostagem, e de $2,4 \mathrm{~W} \mathrm{t}^{-1}$, no material após 60 dias de compostagem, em camadas de material de $1 \mathrm{~m}$ de espessura. Em camadas de espessura de $2 \mathrm{~m}$, a demanda de potência aumentou para 17,7 e $21,7 \mathrm{~W} \mathrm{t}^{-1}$, respectivamente.

\section{REFERÊNCIAS}

COSTA, M.S.S.M.; COSTA, L.A. M.; DECARLI, L. D.; PELÁ, A.; SILVA, C. J.; MATTER, U. F.; OLIBONE, D. Compostagem de resíduos sólidos de frigorífico. Revista Brasileira de Engenharia Agrícola e Ambiental, Campina Grande, v.13, n.1, p.100-107, 2009.

HAUG, R.T. The practical handbook of compost engineering. Boca Raton: Lewis Publishers, 1993. $717 \mathrm{p}$.

INÁCIO, C. de T.; MILLER, P.R.M. Compostagem: ciência e prática para gestão de resíduos orgânicos. Rio de Janeiro: Embrapa Solos, 2009. 156 p. 
JEKAYINFA, S.O. Effect of sirflow rate, moisture content and Ppressure drop on the airflow resistance of locust bean seed. Agricultural Engineering International: the CIGR Journal. Beijing, v.8, 2006. Manuscript FP 06010.

KUSIŃSKA, E. Hydraulic resistance of air flow through wheat grain in bulk. Kom. Mot. Energ. Roln. - Ol Pan, Poland, v.8, p.121-127, 2008.

McGUCKIN, R.L.; EITEMAN, M.A.; DAS, K. Pressure drop through raw food waste compost containing synthetic bulking agents. Journal of Agricultural Engineering Research, New York, v.72, n.4, p.375-384. 1999.

MOREIRA, D.A.; MATOS, A.T.; SARTORI, M.; SILVA, N.C.L.; BARROS, R.T.P.; LUÍS, F.A.R. Perda de carga no fluxo de ar forçado em colunas de material orgânico com diferentes profundidades e estádios de degradação bioquímica. Engenharia na Agricultura, Viçosa-MG, v.16, p.238-247. 2008.

NICOLAI, R.E.; JANI, K.A. Biofilter media mixture ratio of wood chips and compost treating swine odors. Water Science and Technology, Oxford, v.44, n.9, p.261-267, 2001.

ORRICO, A.C.A.; LUCAS JÚNIOR, J.; ORRICO JÚNIOR, M.A.P. Caracterização e biodigestão anaeróbia dos dejetos de caprinos. Engenharia Agrícola, Jaboticabal, v.27, n.3, p.639-647, 2007.

ORRICO JÚNIOR, M.A.P.; ORRICO, A.C.A.; LUCAS JÚNIOR, J. Compostagem da fração sólida da água residuária de suinocultura. Engenharia Agrícola, Jaboticabal, v.29, n.3, p.483-491, 2009.

PEREIRA NETO, J. T. Manual de compostagem, processo de baixo custo - ed. rev. e aum. Viçosa: Ed. UFV, 2007. 81 p.

PINTO, M.T. Higienização de lodos. In: ANDREOLI et al. (Ed.). Lodo de esgotos: tratamento e disposição final. Belo Horizonte: DESA-UFMG/SANEPAR, 2001 p.261-317.

SATISHA, G.C.; DEVARAJAN, L. Effect of amendments on windrow composting of sugar industry pressmud. Waste Management, Amsterdam, v.27, p.1.083-1.091, 2007.

SILVA, N.C.L.; MATOS, A.T.; SARTORI, M.; MOREIRA, D.A.; BARROS, R.T.P.; LUÍS, F.A.R. Variação na pressão estática de ar insuflado em diferentes vazões específicas em colunas de material orgânico com diferentes estádios de degradação bioquímica. Acta Scientiarum Agronomy, Maringá,v.30, p.165-170, 2008.

TORRES, P.; PEREZ, A.; ESCOBAR, J.C.; URIBE, I.E.; IMERY, R. Compostaje de biosólidos de planta de tratamiento de águas residuals. Engenharia Agrícola, Jaboticabal, v.27, n.1, p.267-275, 2007.

ZHU, N. Effect of low initial $\mathrm{C} / \mathrm{N}$ ratio on aerobic composting of swine manure with rice.

Bioresource Technology, Oxford, v.98, n.1, p.9-13, 2007. 